\title{
Paraffin immunofluorescence in the renal pathology laboratory: more than a salvage technique
}

\author{
Nidia C Messias, Patrick D Walker and Christopher P Larsen \\ Nephropath, Little Rock, AR, USA
}

\begin{abstract}
Immunofluorescence studies on paraffin-embedded tissue after Pronase digestion (paraffin immunofluorescence) is used as a salvage technique in renal pathology, when frozen tissue for routine immunofluorescence is inadequate. We have recently found that it is also useful in rare cases in which the immune deposits are 'masked' on routine immunofluorescence, giving false-negative staining by routine immunofluorescence and positive staining by paraffin immunofluorescence. This study aims to evaluate the role of paraffin immunofluorescence in clinical practice with emphasis on its utility to avoid misdiagnosis of cases with masked immune complex deposits. Paraffin immunofluorescence was used in $304(6.1 \%)$ of 4969 native biopsies reviewed from our files. In $207(68.1 \%)$ cases, paraffin immunofluorescence was used as a salvage technique. It was necessary for diagnosis in $24(11.6 \%)$ and had a significant contribution in $63(30.4 \%)$ of these cases. Paraffin immunofluorescence was used to evaluate masked deposits in $97(31.9 \%)$ cases. In $61(62.9 \%)$ of these cases it was used to evaluate masked immune complex glomerular deposits, and in 36 cases (37.1\%) it was used to evaluate masked paraproteins. Of the cases where immune complex deposits were sought, paraffin immunofluorescence was necessary for diagnosis in $16(26.2 \%)$ cases and had a significant contribution in $4(6.6 \%)$ cases. Fourteen of the 20 cases with masked deposits had C3 dominant stain by routine immunofluorescence, which could have been misdiagnosed as C3 glomerulopathy. Overall, paraffin immunofluorescence was necessary or had a significant contribution to diagnosis in $>1 / 3$ of the cases and is a valuable technique in renal pathology.
\end{abstract}

Modern Pathology (2015) 28, 854-860; doi:10.1038/modpathol.2015.1; published online 13 February 2015

Routine processing of a diagnostic renal biopsy includes immunostaining for a battery of immunoglobulin and complement components. ${ }^{1-3}$ Immunofluorescence performed on cryostat sections cut from unfixed frozen, cortical tissue has been used routinely to study renal biopsies for $>50$ years. ${ }^{4}$ The results are critical to the accurate diagnosis of many immunologically mediated glomerular diseases, dysproteinemias and other entities that result from abnormal deposition of proteins in both glomerular and extraglomerular sites. More recently, techniques utilizing formalin-fixed, paraffin-embedded tissue instead have been shown to be nearly as sensitive and specific when cortical tissue for immunofluorescence studies is not available for technical reasons. ${ }^{5,6}$ Fixed tissue used in this way is most

Correspondence: Dr NC Messias, MD, Nephropath, 10180 Executive Center Drive, Suite 100, Little Rock, AR 72211, USA. E-mail: Nidia.messias@nephropath.com

Received 28 October 2014; revised 16 December 2014; accepted 18 December 2014; published online 13 February 2015 commonly used as a salvage technique when inadequate frozen tissue is available for routine immunofluorescence examination and within this setting, immunofluorescence studies done on paraffin-fixed tissue give results quite comparable to those obtained on frozen tissue for most pathogenic immunoglobulins and immunoglobulin fragments, although detection of C3 may be more problematic. ${ }^{6}$ There is also one well-established diagnostic application of this technique outside of the salvage scenario when the addition of a pronasebased antigen retrieval step allows for unmasking of antigenic sites within crystalline inclusions in light chain proximal tubulopathy. ${ }^{7}$ We have found that this same masking phenomenon is occasionally present in glomerular immune complex-type deposits in an entity termed membranous-like glomerulopathy with masked IgG kappa deposits. ${ }^{8}$

The aim of the present study was to extend these findings using tissue available in a large renal biopsy laboratory to determine whether immunofluorescence on pronase-digested paraffin sections 
(paraffin immunofluorescence) would yield additional findings that might alter clinical practice by either enhancing or changing the diagnoses in renal biopsies that had already been studied by routine light, immunofluorescence, and electron microscopy.

\section{Materials and methods}

We reviewed the case files of our institution from January through September of 2013 for all native renal biopsies in which paraffin immunofluorescence had been performed. All cases were processed by light, immunofluorescence, and electron microscopy using routine techniques described below. ${ }^{1}$ All data were collected according to protocols approved by the Schulman Institutional Review Board.

\section{Light Microscopy}

Kidney biopsies were fixed in buffered formalin, dehydrated in graded alcohols, and embedded in paraffin using standard techniques. Serial $3 \mu \mathrm{m}$ thick sections were cut and treated with hematoxylin and eosin, Jones methenamine silver, Masson trichrome, and periodic acid-Schiff reagent.

\section{Immunofluorescence}

For routine immunofluorescence, samples were transported in Michel's media, washed in buffer, and frozen in optimal cutting temperature compound in a cryostat. Sections, cut at $5 \mu \mathrm{m}$, were rinsed in buffer and incubated with fluoresceintagged polyclonal rabbit antihuman antibodies to IgG, IgA, IgM, C3, C4, C1q, fibrinogen, and $\kappa$ - and $\lambda$-light chains (all from Dako, Carpenteria, CA, USA) for $1 \mathrm{~h}$, rinsed, and a coverslip applied using aqueous mounting media. Immunofluorescene stains on pronase-digested sections cut from paraffin-embedded tissue fixed in formalin were performed as deemed necessary according to each individual case. Details of the procedure for paraffin immunofluorescence are described in Table 1. The results for both traditional immunofluorescence and paraffin immunofluorescence were graded on a scale of $0-3$ by trained renal pathologists. The stained slides were evaluated by immunofluorescence microscopy using a Leica L5 filter cube.

\section{Electron Microscopy}

The ends of the renal biopsy specimen were removed as $1 \mathrm{~mm}$ cubes, dehydrated using graded alcohols, and embedded in epon/araldite resin. Sections 1- $\mu \mathrm{m}$ thick were cut using an ultramicrotome, stained with toluidine blue, and examined with a light microscope. Thin sections were examined in a Jeol JEM-1011 electron microscope (Jeol, Tokyo, Japan).
Table 1 Paraffin immunofluorescence procedure

Cut $3 \mathrm{~mm}$ serial sections on organosilane-coated slides.

Oven dry at $37^{\circ} \mathrm{C}$ overnight (or at $60^{\circ} \mathrm{C}$ for $15 \mathrm{~min}$ ).

Deparaffinize: xylene for $10 \mathrm{~min}(2)$, ethanol $100 \%$ for $5 \mathrm{~min}(2)$, $95 \%$ for $5 \mathrm{~min}$.

Wash in distilled water (20 dips).

Rinse in EnVision Flex Wash Buffer (Dako, Carpinteria, CA, USA, Product No. K8007).

Incubate with Proteinase K (Dako, Product No. S302080-2) for $20 \mathrm{~min}$.

Incubate in a wet chamber at $40{ }^{\circ} \mathrm{C}$ for 30 min with antibodies (Dako).

Rinse with PBS $40^{\circ} \mathrm{C}$ for $10 \mathrm{~min}$.

Mount in Vectashield Aqueous Mounting Media.

Examine slides under a dark-field ultraviolet

immunofluorescence microscope.

Photomicrographs were routinely taken at $\times 5000$, $\times 12000$, and $\times 20000$ magnifications.

\section{Study Definitions}

A presumptive diagnosis was given to each case by one of the reviewing pathologists based on the biopsy findings prior to paraffin immunofluorescence results (CL and NM). This presumptive diagnosis was then compared with the final diagnosis assigned to the biopsy after the results of paraffin immunofluorescence were included. The indication for the use of paraffin immunofluorescence and the contribution of this technique were assessed based on comparison between the presumptive diagnosis and the final diagnosis. Indications for paraffin immunofluorescence were divided into two categories: (a) a salvage technique in which the sections on routine immnofluorescence did not contain adequate tissue for diagnosis, and (b) an unmasking technique in cases where the routine immunofluorescence sample was considered adequate. The contribution of paraffin immunofluorescence to the final diagnosis was divided into three categories: (a) did not lead to changes in the diagnosis and/or did not bring any additional information that had an impact to the diagnosis, (b) made a significant contribution to the diagnosis, and (c) was necessary for the diagnosis.

\section{Results}

In total, 4969 native kidney biopsies were examined in our institution from 1 January 2013 through 30 September 2013. Paraffin immunofluorescence was performed on $324(6.5 \%)$ of these biopsies. Glomeruli were present for evaluation in paraffin immunofluorescence sections in $304(6.1 \%)$ cases. Of these 304 cases, 207 (68.1\%) were performed as a salvage technique and the remaining 97 (31.9\%) were performed to look for masked immunoglobulins or light chains. There was no significant difference in age between the groups submitted for 
salvage versus unmasking, with an average age of $52.2 \pm 22.0$ years, and 53.2 \pm 20.4 years, respectively. The results are summarized in Figure 1.

\section{Paraffin Immunofluorescence used as a Salvage Technique}

Of the 207 cases in which paraffin immunofluorescence was performed as a salvage technique, $183 \mathrm{did}$ not have any intact glomeruli on initial routine immunofluorescence evaluation. Twenty cases were performed as an adjunct to immunofluorescence on frozen tissue, because only a minimal $(<3)$ number of glomeruli was present in the sections or artifactual changes made them unsuitable for evaluation, and four cases were performed to evaluate light chain casts and amyloid deposits, which were not present in tissue submitted for routine immunofluorescence. Paraffin immunofluorescence did not result in changes to the diagnosis in 120 cases (58\%), made a significant contribution to the diagnosis in $63(30.4 \%)$, and was necessary for the diagnosis in 24 cases $(11.6 \%)$. Among the 24 cases in which salvage paraffin immunofluorescence was considered necessary, the most frequent diagnosis was IgA nephropathy (15 cases) followed by membranous glomerulopathy (4 cases). There were 12 cases of membranous glomerulopathy in which it was determined that the paraffin immunofluorescence made a significant contribution, as the light and electron microscopy findings in membranous glomerulopathy are fairly specific for disease. The four cases of membranous glomerulopathy in which paraffin immunofluorescence was judged to be necessary were a result of additional limitations such as a lack of glomeruli for electron microscopy. The remaining cases consisted of immune complex- mediated glomerulonephritis, not otherwise specified (two cases), C1q nephropathy (one), C3 glomerulopathy (one), and class I lupus nephritis (one).

\section{Paraffin Immunofluorescence used for Antigen Unmasking}

The 97 cases in which paraffin immunofluorescence was used to rule out masked deposits were divided into two subgroups: unmasking of light chain deposits to evaluate for light chain-associated disease $(n=36,37.1 \%)$ and unmasking of immunoglobulins to evaluate immune complex-mediated disease $(n=61,62.8 \%)$.

In the 36 cases studied by paraffin immunofluorescence to evaluate light chain-associated diseases including amyloidosis, light chain cast nephropathy, light chain proximal tubulopathy, and light chain-deposition disease, paraffin immunofluorescence made a significant contribution to the diagnosis in only two (5.5\%). One case was light chaindeposition disease combined with light chain proximal tubulopathy in which the light chain proximal tubulopathy had not been identified by routine immunofluorescence. In a second case, paraffin immunofluorescence was considered necessary for the diagnosis of light chain proximal tubulopathy with crystals. In this case, the light chain crystals did not show any staining by routine immunofluorescence but were positive by paraffin immunofluorescence. There were no cases of masked paraprotein in any other forms of light chain-associated disease such as amyloidosis, light chain cast nephropathy, or light chain deposition disease.

In 61 cases paraffin immunofluorescence was performed to look for masked immunoglobulin

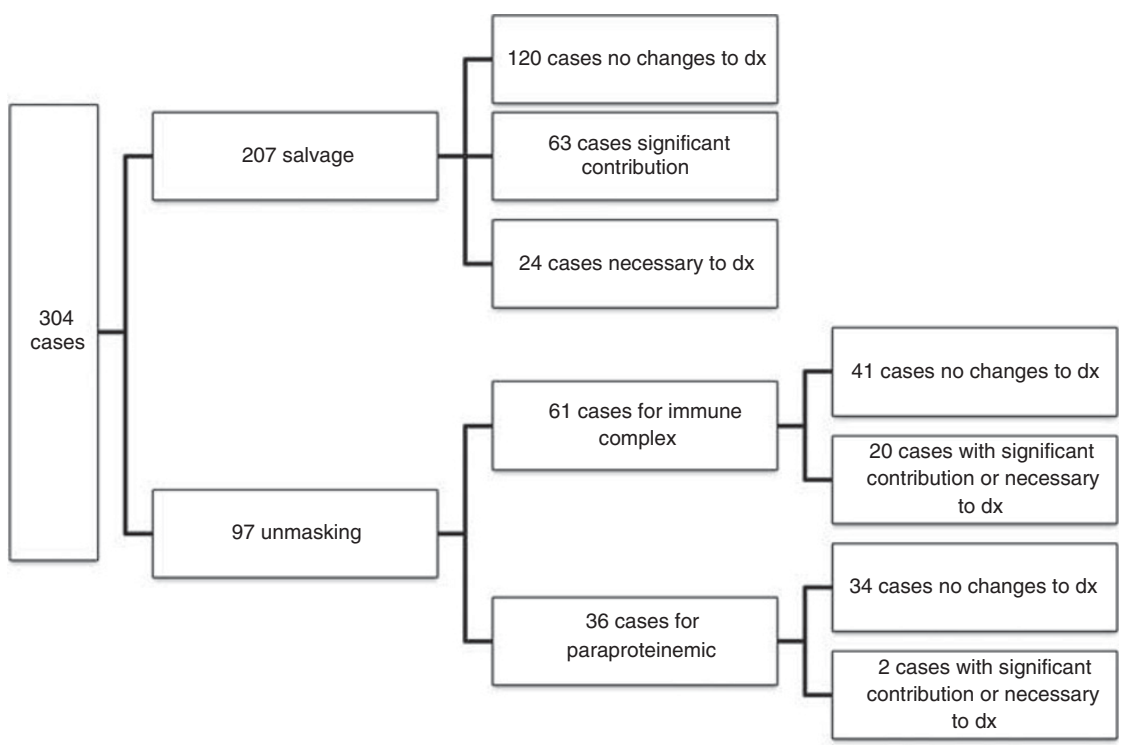

Figure 1 Distribution of paraffin immunofluorescence cases according to indication and contribution to results. 
Table 2 Findings on cases with masked deposits

\begin{tabular}{|c|c|c|c|c|c|c|c|c|c|c|c|c|c|c|c|c|c|c|c|}
\hline \multirow[b]{2}{*}{$\#$} & \multirow[b]{2}{*}{ Age } & \multicolumn{7}{|c|}{ Routine immunofluorescence ${ }^{\mathrm{a}}$} & \multicolumn{7}{|c|}{ Paraffin immunofluorescence ${ }^{\mathrm{a}}$} & \multirow[b]{2}{*}{ Diagnosis before PIF } & \multirow[b]{2}{*}{ Final diagnosis } & \multicolumn{2}{|c|}{ EM deposits } \\
\hline & & $\operatorname{Ig} A$ & $\operatorname{Ig} G$ & $\operatorname{Ig} M$ & C3 & $C 1 q$ & K & $\lambda$ & $\operatorname{Ig} A$ & $\operatorname{Ig} G$ & $\operatorname{Ig} M$ & C3 & $C 1 q$ & K & $\lambda$ & & & Subend & Subep \\
\hline 1 & 32 & 0 & 0 & 1 & 1 & 0 & 0 & 0 & 0 & 3 & 1 & 2 & NP & 3 & 0 & Crescentic GN, pauci immune & MGMIGK & 0 & + \\
\hline 2 & 15 & 0 & 0 & 0 & 1 & 0 & 0 & 0 & 0 & 3 & 0 & NP & NP & 3 & 0 & C3 GN, epimembranous & MGMIGK & 0 & + \\
\hline 3 & 37 & 0 & 1 & 0 & 2 & 0 & 1 & 0 & 0 & 3 & 0 & 3 & NP & 3 & 0 & C3 GN, epimembranous & MGMIGK & 0 & + \\
\hline 4 & 22 & 0 & 0 & 0 & 0 & 0 & 0 & 0 & 0 & 2 & 0 & 2 & 2 & 2 & 0 & Crescentic GN, pauci immune & MGMIGK & + & + \\
\hline 5 & 20 & 0 & 0 & 0 & 2 & 0 & 0 & 0 & 0 & 3 & 0 & NP & NP & 3 & 0 & C3 GN, epimembranous & MGMIGK & 0 & + \\
\hline 6 & 19 & 0 & 0 & 0 & 1 & 0 & 0 & 0 & 0 & 3 & 0 & NP & NP & 3 & 0 & C3 GN, epimembranous & MGMIGK & 0 & + \\
\hline 7 & 18 & 0 & 0 & 0 & 3 & 0 & 1 & 0 & NP & 3 & NP & NP & NP & 2 & 1 & C3 GN, epimembranous & MGMIGK & 0 & + \\
\hline 8 & 55 & 0 & 0 & 0 & 2 & 0 & 0 & 0 & 0 & 3 & 0 & 3 & 0 & 3 & 0 & C3 GN, epimembranous & MGMIGK & + & + \\
\hline 9 & 16 & 0 & 0 & 0 & 2 & 0 & 0 & 0 & NP & 3 & NP & NP & NP & 3 & 0 & C3 GN, epimembranous & MGMIGK & 0 & + \\
\hline 10 & 40 & 0 & 0 & 0 & 3 & 0 & 0 & 0 & 0 & 0 & 3 & 3 & NP & 3 & 0 & MPGN c/w C3 GN & MPGN with monoclonal Ig & + & + \\
\hline 11 & 66 & 0 & 0 & 0 & 1 & 0 & 0 & 0 & 1 & 0 & 3 & 2 & NP & 0 & 3 & MPGN c/w C3 GN & MPGN with monoclonal Ig & + & + \\
\hline 12 & 64 & 0 & 0 & 0 & 3 & 0 & 0 & 0 & 0 & 2 & 0 & NP & NP & 2 & 0 & MPGN c/w C3 GN & MPGN with monoclonal Ig & + & + \\
\hline 13 & 57 & 0 & 0 & 0 & 0 & 0 & 0 & 0 & 0 & 3 & 1 & 3 & NP & 3 & 0 & Pauci immune MPGN & MPGN with monoclonal Ig & + & 0 \\
\hline 14 & 83 & 0 & 0 & 0 & 0 & 0 & 0 & 0 & 0 & 2 & 3 & NP & NP & 3 & 2 & MPGN c/w chronic TMA & MPGN c/w ME cryo & + & 0 \\
\hline 15 & 62 & 0 & 0 & 1 & 2 & 0 & 1 & 0 & 0 & 3 & 3 & NP & NP & 2 & 3 & MPGN c/w C3 GN & MPGN c/w ME cryo & + & + \\
\hline 16 & 55 & 0 & 1 & 1 & 3 & 0 & 1 & 1 & 0 & 2 & 3 & NP & NP & NP & NP & MPGN c/w C3 GN & MPGN c/w ME cryo & + & + \\
\hline 17 & 55 & 0 & 0 & 2 & 3 & 0 & 1 & 1 & 0 & 3 & 3 & 3 & 0 & 3 & 2 & MPGN c/w C3 GN & MPGN c/w ME cryo & + & 0 \\
\hline 18 & 74 & 0 & 0 & 1 & 2 & 0 & 1 & 1 & 0 & 2 & 2 & 2 & 0 & 3 & 3 & MPGN c/w C3 GN & MPGN c/w ME cryo & + & 0 \\
\hline 19 & 63 & 0 & 1 & 0 & 0 & 0 & 1 & 1 & 0 & 2 & 1 & 2 & NP & 2 & 1 & MPGN & MPGN c/w ME cryo & + & 0 \\
\hline 20 & 80 & 0 & 0 & 2 & 0 & 0 & 2 & 0 & 0 & 3 & 2 & 0 & NP & 3 & 1 & MPGN c/w type1 cryo & MPGN c/w ME cryo & + & 0 \\
\hline
\end{tabular}

Abbreviations: C3 GN, C3 glomerulonephritis; Cryo, cryoglobulinemic glomerulonephritis; c/w, consistent with; EM, electron microscopy; IF, immunofluorescence; ME, mixed essential; MGMIGK, membranous-like glomerulonephritis with masked IgG kappa deposits; MPGN, membranoproliferative glomerulonephritis; NP, not performed; PIF, paraffin immunofluorescence; Subend, subendothelial; Subep, subepithelial. ammunofluorescence intensities scored on 0-3 scale.

deposits in immune complex-type diseases. All of these cases contained an adequate number of glomeruli on initial routine immunofluorescence evaluation. Paraffin immunofluorescence did not result in significant changes to the diagnosis in 41 cases $(67.2 \%)$ because results obtained with paraffin immunofluorescence were the same as routine immunofluorescence evaluation.

However, there were 20 cases $(26.2 \%$ in this group) in which paraffin immunofluorescence was necessary to reach the correct diagnosis (Table 2 and Figure 2) and 4 cases (6.6\%) in which paraffin immunofluorescence made a significant contribution to the diagnosis, by enhancing the results of immunofluorescence, and corroborating observations from light microscopy and EM (Table 2). Light microscopy findings on these cases that were either necessary or had an important contribution to the diagnosis showed: a membranoproliferative pattern $(n=11,55 \%)$, a membranous pattern $(n=7,35 \%)$, and crescentic glomerulonephritis $(n=2,10 \%)$. Six of the cases of membranous-like glomerulopathy with masked IgG kappa deposits were previously reported. ${ }^{8}$ Among the seven cases consistent with mixed essential cryoglobulinemic glomerulonephritis on final diagnosis, there were three patients positive for hepatitis C virus, three with Sjögren's disease, and one with idiopathic cryoglobulinemia. Ultrastructural studies of the 20 cases in which paraffin immunofluorescence was necessary or important for diagnosis revealed mesangial deposits in all the cases in which paraffin immunofluorescence showed masked deposits. Furthermore, in addition to the mesangial deposits, all of these 20 cases had subendothelial or subepithelial deposits, or a combination of both (Table 2).

\section{Discussion}

We detail our use of paraffin immunofluorescence in a large renal biopsy laboratory and find that it is useful as more than a salvage technique. In fact, in many cases it was crucial to arriving at the proper diagnosis when false-negative staining was present by routine immunofluorescence. Though the use of paraffin immunofluorescence is currently uncommon, it is important to maintain a high index of suspicion for the possibility of masked deposits given the potential of misdiagnosis if they are not detected.

Many of the cases in our series would meet diagnostic criteria for C3 glomerulonephritis if only routine immunofluorescence was performed and the masked deposits had not been recognized. ${ }^{9,10}$ This mistake would likely lead to unnecessary laboratory evaluation and potential mismanagement of the disease. We have recently described an entity termed membranous-like glomerulopathy with masked IgG kappa deposits. This form of glomerulonephritis primarily affects young females who often have other vague autoimmune phenomenon. ${ }^{8}$ We know of no other reports of masked immune complex-type deposits, which require antigen retrieval to elicit a staining reaction. However, there is one case report of 'monoclonal gammopathy-associated MPGN' in which the IgG 

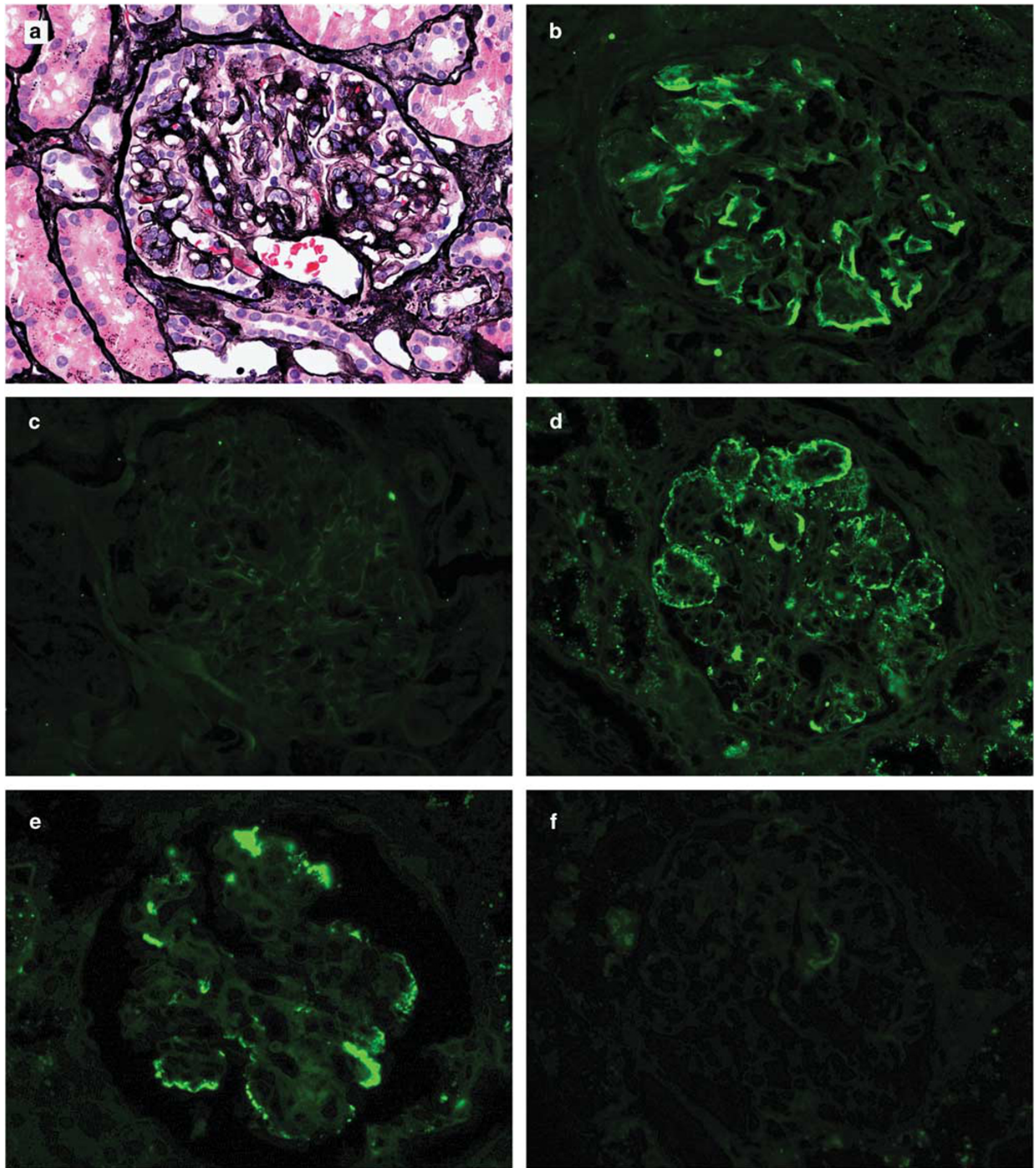

Figure 2 Light and immunofluorescence microscopy findings in a case of membranoproliferative glomerulonephritis with masked deposits (case 12 from Table 2). (a) Glomerulus with mesangial expansion and extensive basement membrane duplication (Jones methenamine silver; original magnification $\times 400$ ). (b) Positive staining for C3 on routine immunofluorescence (direct immunofluorescence). (c) IgG (shown here) as well as other immunoglobulins and light chains were negative within glomeruli by routine immunofluorescence (direct immunofluorescence). (d) Positive IgG staining within a glomerulus in the paraffin-embedded tissue after pronase digestion (direct immunofluorescence). (e) Kappa was also diffusely positive within glomeruli by paraffin immunofluorescence (direct immunofluorescence). (f) Negative staining for lambda light chains by paraffin immunofluorescence (direct immunofluorescence).

kappa deposits were apparently masked by routine immunofluorescence and detected utilizing laser microdissection-mass spectrometry. ${ }^{11}$ This is another example of false-negative staining by routine immunofluorescence, which was detected utilizing a more sophisticated technique. The authors use this 
report to highlight the utility of mass spectrometry as a tool for the evaluation of glomerulonephritis. In that study, paraffin immunofluorescence was not used to detect masked deposits but would be a significantly simpler alternative to mass spectrometry.

Paraffin immunofluorescence is clearly not feasible or necessary to perform in the vast majority of renal biopsy cases. However, there are specific situations, which should trigger consideration of performing this study. Paraffin immunofluorescence is particularly important in cases where the initial diagnosis is C3 glomerulonephritis. We routinely perform this technique on biopsies that show C3only staining and either a membranoproliferative or membranous-like pattern. It is also useful in cases that show immune complex-type deposits by electron microscopy with little to no staining by immunofluorescence or when the staining does not fit the expected pattern (e.g., HCV-associated cryoglobulinemic glomerulonephritis without immunoglobulin staining). In general, if there is something unexpected about the immunofluorescence staining pattern or the immunofluorescence and EM findings are not confirmatory then strong consideration should be given to using paraffin immunofluorescence.

The paraffin immunofluorescence technique is simple to perform for renal pathology laboratories familiar with routine immunofluorescence. Although the interpretation is similar to routine immunofluorescence, there are important pitfalls to be aware of. Owing to fixation, the glomerular capillaries will often contain residual serum on paraffin immunofluorescence, which is not typically present in routine immunofluorescence sections from frozen tissue. This serum will stain nonspecifically positive for many of the antibodies used (see Figure 3). Therefore, it is important to pay close attention to which area of the glomerulus is

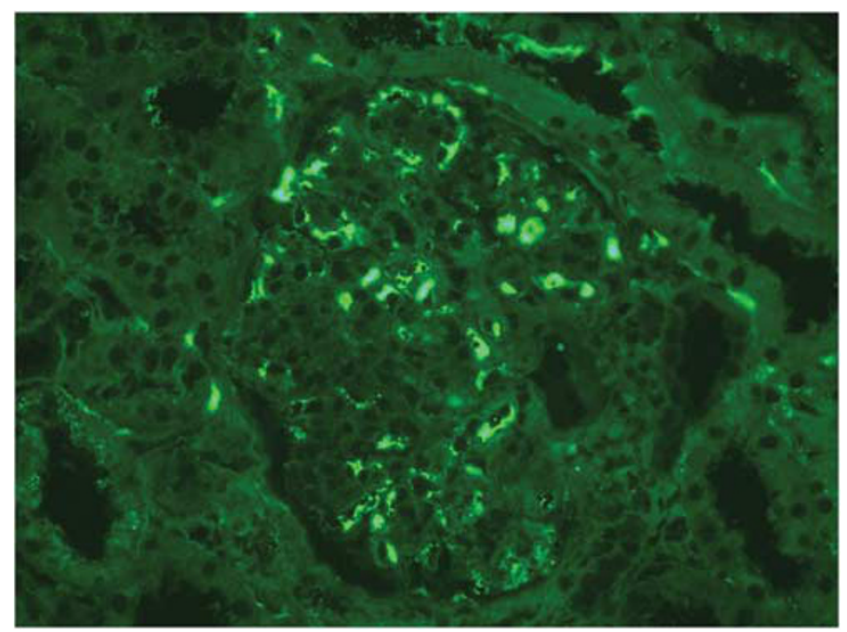

Figure 3 Potential diagnostic pitfall of paraffin immunofluorescence method. IgA staining is strongly positive within the capillary spaces of a glomerulus by paraffin immunofluorescence. The location is the key to avoid misdiagnosing this artifact as positive staining. staining to ensure that this intracapillary staining is not mistaken for immune complex deposition. Another potential pitfall to be aware of is that paraffin immunofluorescence is not a sensitive technique for the detection of C3. Although it can be used for C3 staining, it is our experience and has been previously reported that C3 stains considerably weaker by paraffin immunofluorescence. ${ }^{6}$ An additional potential pitfall that is important to be aware of with this technique is in the evaluation for anti-glomerular basement membrane disease. Paraffin immunofluorescence rarely demonstrates the linear IgG deposits and should not be used for this purpose. ${ }^{6}$ At last, it is important to point out that the paraffin immunofluorescence technique we are describing is based on our experience in formalin-fixed tissue. The performance has not been tested with other fixatives such as Bouin solution. On the basis of these pitfalls we believe that routine immunofluorescence should be considered a secondary analysis best used as a salvage technique or to increase the sensitivity for the detection of immunoglobulin in the specific situations described herein. We do not maintain that it should supplant routine immunofluorescence in the evaluation of renal biopsy specimens.

The reason why some glomerular deposits stain on paraffin and not routine immunofluorescence is a mystery at this time. Perhaps the absence of covalent bonds between proteins in the tissue resulting from formalin cross-linking allows the immunoglobulin to be lost in the wash steps of routine immunofluorescence tissue before analysis. It is also possible that there is a characteristic of the immunoglobulin deposited in the tissue in these masked cases that render it resistant to antibody binding in the absence of a retrieval step. This could be a quaternary arrangement of the immunoglobulin or a charge-charge interaction between the antibody implored and the immunoglobulin in the tissue that hinders antibody binding in the absence of retrieval. At this point in time we only know that the antigenic epitopes are not available for antibody binding in some cases of routine immunofluorescence.

Immunofluorescence on formalin-fixed paraffinembedded tissue after pronase digestion is a valuable technique for renal pathology laboratories. Our results confirm previous studies that document its utility as a salvage technique when insufficient tissue is available for routine immunofluorescence. ${ }^{5}$ But most importantly, we describe a second important use, which is the prevention of a misdiagnosis due to masked immune complex-type deposits.

\section{Acknowledgments}

We would like to thank Dr William Couser for reviewing the manuscript and our colleagues at Nephropath for their input in the study. 


\section{Disclosure/conflict of interest}

The authors declare no conflict of interest.

\section{References}

1 Walker PD, Cavallo T, Bonsib SM. Practice guidelines for the renal biopsy. Mod Pathol 2004;17:1555-1563.

2 Walker PD. The renal biopsy. Arch Pathol Lab Med 2009;133:181-188.

3 Pullman JM, Ferrario F, Nast CC. Actual practices in nephropathology: a survey and comparison with best practices. Adv Anat Pathol 2007;14:132-140.

4 Coons AH, Kaplan MH. Localization of antigen in tissue cells. J Exp Med 1950;91:1-13.

5 Molne J, Breimer ME, Svalander C. Immunoperoxidase versus immunofluorescence in the assessment of human renal biopsies. Am J Kidney Dis 2005;45:674-683.

6 Nasr SH, Galgano SJ, Markowitz GS, et al. Immunofluorescence on pronase-digested paraffin sections: a valuable salvage technique for renal biopsies. Kidney Int 2006;70:2148-2151.

7 Larsen CP, Bell JM, Harris AA, et al. The morphologic spectrum and clinical significance of light chain proximal tubulopathy with and without crystal formation. Mod Pathol 2011;24:1462-1469.

8 Larsen CP, Ambuzs JM, Bonsib SM, et al. Membranouslike glomerulopathy with masked IgG kappa deposits. Kidney Int 2014;86:154-161.

9 Pickering MC, D’Agati VD, Nester CM, et al. C3 glomerulopathy: consensus report. Kidney Int 2013;84: 1079-1089.

10 Servais A, Frémeaux-Bacchi V, Lequintrec M, et al. Primary glomerulonephritis with isolated C3 deposits: a new entity which shares common genetic risk factors with hemolytic uremic syndrome. J Med Genet 2007; 44:193-199.

11 Jain D, Green JA, Bastacky S, et al. Membranoproliferative glomerulonephritis: the role for laser microdissection and mass spectrometry. Am J Kidney Dis 2014;63:324-328. 